\title{
The High Energy cosmic-Radiation Detection (HERD) facility on board the Chinese Space Station: hunting for high-energy cosmic rays
}

\author{
F. Gargano ${ }^{a, *}$ \\ on behalf of the HERD collaboration \\ (a complete list of authors can be found at the end of the proceedings) \\ ${ }^{a}$ Istituto Nazionale di Fisica Nucleare (INFN) - Sezione di Bari \\ Via Orabona 4, 70126, Bari, Italy \\ E-mail: fabio.gargano@ba.infn.it
}

The High Energy cosmic-Radiation Detection (HERD) facility has been proposed as a space astronomy payload onboard the future China's Space Station (CSS) aimed to detect charged cosmic-rays and gamma-rays from few $\mathrm{GeV}$ to $\mathrm{PeV}$ energies. The main science objectives of HERD are searching for dark matter particles, the study of cosmic ray chemical composition and high energy gamma-ray observations. HERD will extend high precision and high statistics spectral measurements of individual cosmic ray species up to few $\mathrm{PeV}$, reaching the knee of the all-particle spectrum. It will also observe the gamma-ray sky from a few hundred of $\mathrm{MeV}$ up to $1 \mathrm{TeV}$ contributing to multi-messenger astronomy together with ground-based high energy gamma-ray telescope and neutrino and gravitational waves detectors.

$37^{\text {th }}$ International Cosmic Ray Conference (ICRC 2021)

July 12th - 23rd, 2021

Online - Berlin, Germany 


\section{Introduction}

It is widely accepted that neutral, cold/warm and non-baryonic dark matter (DM) dominates the total matter content in the universe. One way to detect DM is to search in space for its annihilation/decay products, which may lead to characteristic features in the observed spectra of cosmic electrons (plus positron) or gamma-ray spectra. Some circumstantial evidence or hints of anomalies have been reported; however, astrophysical sources like pulsars and pulsar wind nebulae can also contribute to these results. Future more precise measurements at higher energies and $e^{+}+e^{-}$anisotropy observation are still needed to probe the origin of high energy electrons.

The steepening of the primary cosmic ray (CR) spectrum around PeV, the so-called "knee" region, is a classic but still unresolved problem in CR physics since the first observations in 1958.

Ground-based extensive air shower experiments have difficulties in making compositionresolved high-energy resolution measurements of the fine structure of the "knee". Space experiments, which can measure the particle energy and charge directly, have been suffering from small geometrical factor and limited energy range to make statistically meaningful measurements of the "knee".

Several generations of wide field of view (FOV) space gamma-ray telescopes in the $\mathrm{GeV}$ energy regime and ground based narrow FOV gamma-ray telescopes in hundreds of GeV energy regimes have discovered several new populations of extreme astrophysical objects, which allow deeper understanding of the laws of nature under extreme physical conditions only available in cosmic laboratories. In particular the wide FOV space gamma-ray telescopes often provide crucial guidance to the observations of the ground-based narrow FOV telescopes. Unfortunately, the much more powerful ground-based Cherenkov Telescope Array (CTA) currently under development may not have the much needed guidance from a space wide FOV gamma-ray telescope, once the Fermi satellite stops operations. A new wide FOV space gamma-ray telescope is urgently needed to replace Fermi.

In order to address the above major problems in fundamental physics and astrophysics, the High Energy cosmic-Radiation Detection (HERD) facility has been proposed as one of several space astronomy payloads onboard the future China's Space Station (CSS), which is planned for operation starting around 2027 for about 10 years.

The primary scientific objectives of HERD are: to search for signatures of the annihilation/decay products of dark matter particles in the energy spectra and anisotropy of high energy electrons from $10 \mathrm{GeV}$ to $100 \mathrm{TeV}$ and in the gamma-ray spectrum from $500 \mathrm{MeV}$ to $100 \mathrm{TeV}$; to measure precisely the energy spectra and composition of primary cosmic rays from $30 \mathrm{GeV}$ up to $\mathrm{PeV}$ in order to determine the mechanism of the cosmic rays 'knee' structure. The secondary scientific objectives of HERD include wide FOV monitoring of the high energy gamma-ray sky from $100 \mathrm{MeV}$ for gamma-ray bursts, active galactic nuclei and Galactic microquasars [1].

\section{Scientific Objectives}

\subsection{Measurement of cosmic electron spectrum and Dark Matter signal Search}

Numerous astrophysical and cosmological observations have shown that the normal baryonic matter only occupies $\sim 15 \%$ of the matter component in the universe, while the faction of dark 
matter (DM) is $\sim 85 \%$. The observations indicate that DM particles should be stable, neutral, and non-relativistic. However, the microscopic properties of DM particles remains unclear. Since the standard model of the particle physics cannot provide a suitable DM candidate, the interpretation of DM requires new physics beyond the standard model itself. Therefore, the detection of DM signals is of great interest to both the particle physics and astrophysics communities.

A popular DM candidate is the so-called weakly interacting massive particle (WIMP). In general, there are three kinds of detection experiments for WIMP, namely collider detection, direct detection, and indirect detection. The collider detection searches for the DM signals in the experiments, generally looking for DM signature in events with large missing energy and momentum. The direct detection probes the scattering signal induced by DM particles in the detector, while the indirect detection searches for the DM annihilation or decay products in high energy cosmic-rays (CRs). The future observations of HERD will be able to detect the possible DM signals in high energy CR electrons/positrons and gamma-rays spectra.

Positron excess, compared with the ordinary expectation of the CR physics, in the CR flux have been reported by PAMELA and very precisely measured by AMS-02 in an extended energy range up to $700 \mathrm{GeV}$. A variety of theoretical studies focusing on the DM interpretation of this excess have been performed in the literature, however the origin of these high energy positrons remain debatable.

In order to distinguish the origin of the high energy electrons/positrons, either from DM annihilation or from astrophysical sources, the observed electron-positron spectrum at higher precision and higher energy scales is very important. The Chinese satellite experiment DAMPE, which was launched in 2015, can precisely measure the electron-positron spectrum up to $10 \mathrm{TeV}$ [2]. In 2017, the DAMPE collaboration reported their first result of CR electrons/positrons, which shows some interesting spectral features at $\sim \mathrm{TeV}$. Compared with DAMPE, the HERD experiment has a larger acceptance, and can measure the electron-positron spectrum with higher precision and at higher energy. Therefore, the future HERD measurement of CR electrons/positrons will be important for the high energy astrophysics and DM studies.

As shown in the literature [3, 4] the AMS-02 data can be explained by both astrophysical and DM sources. The recent DAMPE result in the electron plus positron spectrum shows a break at $0.9 \mathrm{TeV}$ [2], that could be explained with energy limit of the background cosmic electron spectrum [5]. Therefore, the cutoff of the positron spectrum has to be below the break energy at $\sim 0.9 \mathrm{TeV}$ as seems to be favoured in the recent AMS-02 results. It has long been believed that shape of the energy spectrum cutoff is different for the astrophysical source and DM annihilation. The former is generally believed to have an exponential cutoff, which is softer than the hard cutoff by DM annihilation when near the DM mass. A possible way to distinguish the two kinds of shape at the cutoff is to measure the fine structure at the electron plus positron spectrum with a high precision instrument, like HERD.

In Fig. 1 we show the HERD possibility to distinguish the electron plus positron spectra in the DM annihilation and nearby pulsars scenarios. As shown in the figure it is clear that the two spectra can by clearly distinguished by 1 year data accumulation at HERD. Once the 'kink' can be identified as the positron spectrum cutoff the nature of DM particles can be well determined. 


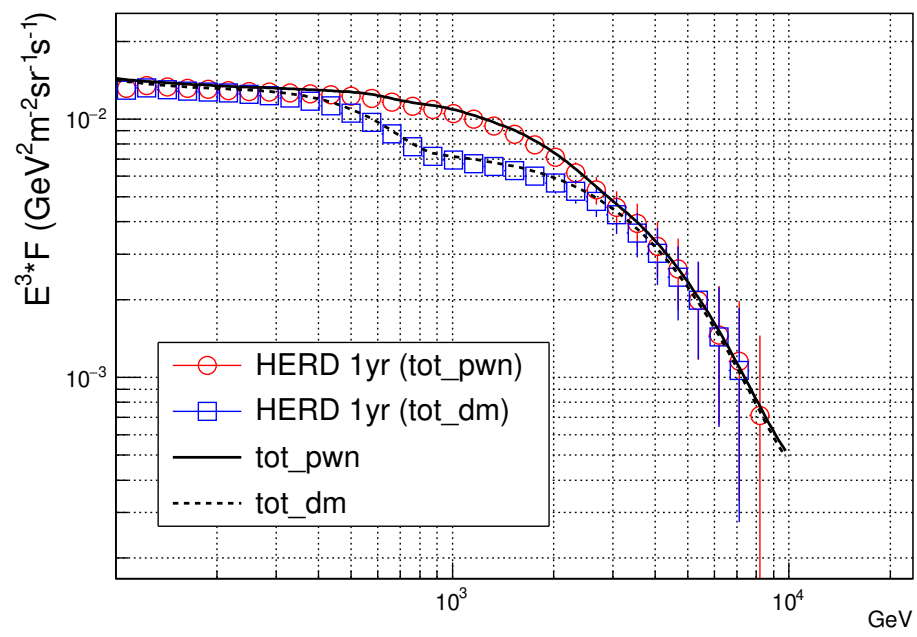

Figure 1: Simulation of HERD capability to distinguish the total electron plus positron spectra in two different scenarios. With one year data accumulation HERD can clearly distinguish between a smooth spectrum (pulsar) or a spectrum with a 'kink' (DM).

\subsection{The contribution to CR electrons from nearby astrophysical sources}

The positron excess observed by PAMELA and AMS-02 has stimulated a lot of interests and many theoretical papers on the origin of the excess positrons have been published. According to these studies, the explantion are attributed to two kinds, that is, the DM annihilation and the nearby pulsars, both of which can contribute positron/electron pairs. The excess positron and electron pairs as well as the primary CR electron background can explain the data precisely[3,4]. Therefore, to determine the source of the excess positrons we need to measure the electron spectrum up to higher energies [6].

An important feature for electrons is that during the propagation process of electron/positron, high energy CR electrons would quickly lose their energies with a large rate, which is proportional to $E^{2}$. Consequently, the observed $\mathrm{CR}$ electrons above $\sim \mathrm{TeV}$ are dominantly contributed by the sources located within $\sim 1 \mathrm{kpc}$ around the solar system, due to the small propagation distance of high energy electrons. Since the HERD experiment can measure the electron-positron spectrum up to $\sim 10 \mathrm{TeV}$ with high precision, the significant contribution from nearby astrophysical sources within $\sim 1 \mathrm{kpc}$ is expected to be detected at HERD.

\subsection{The measurement of the anisotropy of CR electrons}

Since charged CR particles are deflected from their original directions by the Galactic magnetic field, the locations of CR sources cannot be directly determined by experiments. However, if a single source contribute to the observed CR electrons/positrons significantly, the electrons/positrons from the direction of this source are more than those from the opposite direction, and can induce a measurable anisotropy. On the other hand, the contributions to electrons/positrons from DM are almost isotropic due to the flat DM density distribution near the solar system. Therefore, an 
observed anisotropy of electron/positron flux will be a strong evidence for the astrophysical origin of high energy electrons/positrons.

The HERD instrument has a very large acceptance, and will collect a large amount of CR electron/positron events during a long operating period. Therefore, for the aim of distinguishing the origins of high energy electrons/positrons from DM and astrophysical sources, the most important advantage of HERD is the anisotropy measurement.

\section{Origin of Cosmic Rays}

It has been more than one hundred years since the first discovery of cosmic rays (CRs) in 1910s. CRs played very important roles in exploring the fundamental particle physics for nearly a half century before powerful accelerators were widely applied for such studies. Nowadays, the study of CRs has entered another golden era, due mainly to their connection with 1) physics at the highest energy end, 2) indirect search for particle dark matter, and 3) site and mechanism of astrophysical accelerators. The highest energy particle ever recorded has an energy of $\sim 3 \times 10^{20}$ $\mathrm{eV}$ [7], which is comparable to the kinetic energy of a macroscopic object and is much higher (by several orders of magnitude) than the maximum achievable energy of man-made accelerators. CRs are a unique probe to test the known physics laws at this very high energy limits. In addition, the nature of the mystery dark matter, which occupies $\sim 1 / 4$ of the Universe's density content and is 5 times more abundant than the baryonic matter, is another big question of modern physics (see the previous section). Finally, many questions about CRs themselves are also very interesting, such as "where are they produced?", "how are they accelerated to such high energies?", "how do they propagate in the Galaxy and the Universe?".

Several theoretical solutions have been proposed, exploiting different hypotheses on source properties/populations, acceleration/propagation mechanisms and particle physics issues at high energies, in order to give a satisfactory explanation of the so-called knee in the all-particle energy spectrum at about $3 \mathrm{PeV}[8]$.

In particular, it remains unsolved whether the (dominant) origin of the knee is due to the reach of the maximum energy achievable at the source or to diffusion processes in the Galaxy. In both cases a rigidity dependent sequence of knees in each single element spectrum is the most probable scenario [9].

For the analysis of the CR flux, direct measurements carried on space or stratospheric balloons actually give the best performance in terms of both energy resolution and charge identification. However, due to their limited acceptance and the steeply falling fluxes, they could hardly reach, up to now, energies of hundreds of $\mathrm{TeV}$ and they did not yet give clear information on the steepening of various elements nor on the knee of each species or of the all-particle spectrum itself $[10,11]$.

Current data suggest a hardening of the spectra above about $0.2 \mathrm{TeV} /$ nucleon and spectral indices $\gamma$ (above that energy) of about -2.6 for all considered elements but for protons, that show a softer spectrum with $\gamma \simeq-2.7[12,13]$. On the other hand, indirect measurements explore the relevant energy region through the detection of Extensive Air Showers (EAS) in the atmosphere, but with unavoidable large systematics (and worse resolutions) due to detector sampling, showerto-shower fluctuations and our limited knowledge of hadronic interactions at these energies, mainly in the very forward region [8, 14]. Moreover they cannot reach full efficiency for all nuclear species 


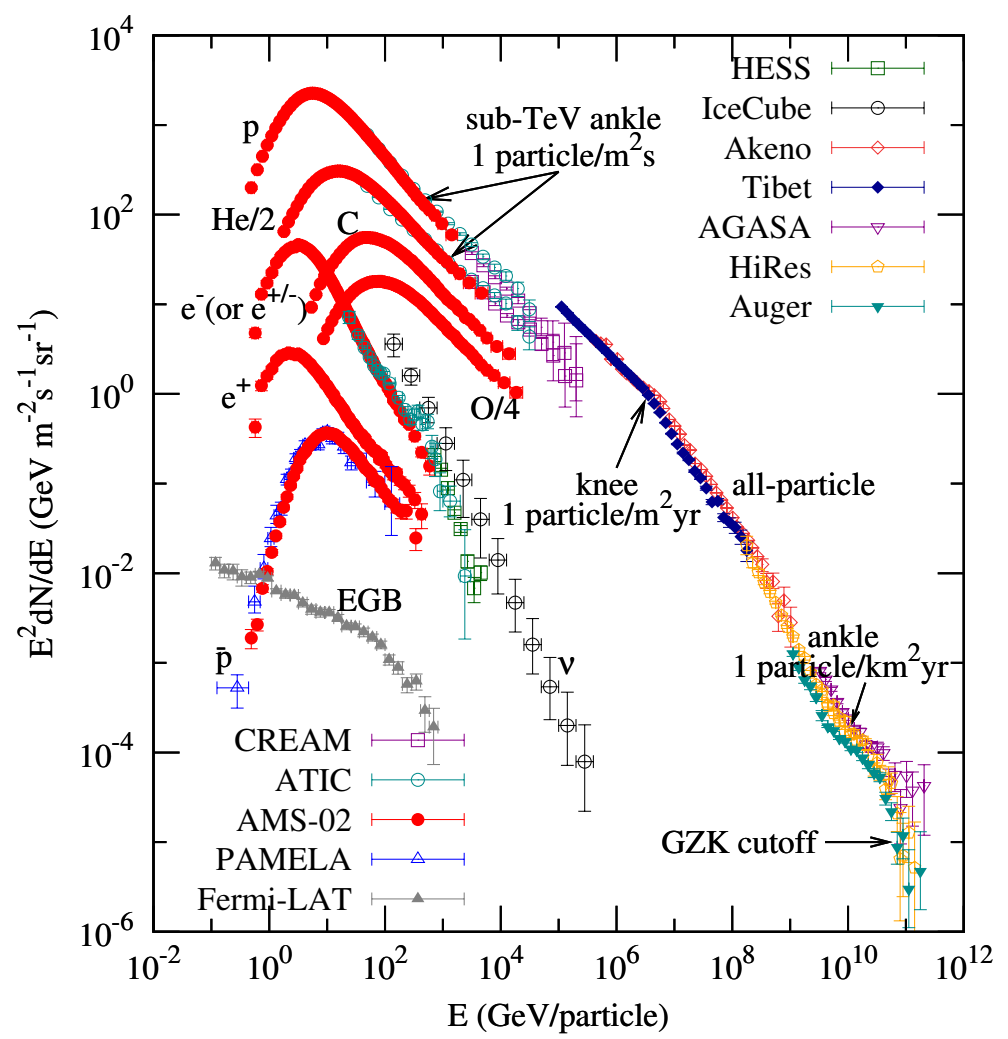

Figure 2: Up-to-date summary of measurements of CR energy spectra. For energies below $\sim 100 \mathrm{TeV}$, the spectra of different species are shown, and for higher energies the all-particle spectra are plotted. References of data: CREAM; ATIC; AMS-02; PAMELA; Fermi-LAT; HESS; IceCube; Akeno; Tibet;AGASA; HiRes; Auger;

below few hundred $\mathrm{TeV}$, even for high altitude locations. For light elements their threshold can be lowered to a few $\mathrm{TeV}$, showing interesting hints for a steepening of the proton and helium spectra just below $1 \mathrm{PeV}[15]$.

It is then mandatory to explore the sub-PeV region with high precision direct measurements in order to study the energy spectra of each individual nuclear species, to measure the various spectral indices, to detect any possible hardening and to set mass composition below the knee of the all-particle flux. If possible the detection of the steepening of each single species and then the explanation of the all-particle knee would be a crucial result in understanding Galactic CR physics, also serving as fundamental input to the study of the extra-galactic component.

\subsection{Potential of HERD}

The unique design of HERD together with the unprecedented depth of the calorimeter and the high resolution tracker, will allow the extension of high precision measurements on proton and nuclei spectra up to the highest energies, even beyond $1 \mathrm{PeV}$. Moreover a clear identification of each nuclear species will be possible through the plastic scintillator detector, the analog readout of the silicon tracker and by the calorimeter itself. Energy resolution for the electromagnetic and hadronic 
showers will be at the $1 \%$ and $30 \%$ level, respectively. A detailed study of the high energy photon flux and indirect signal of dark matter will also be possible.

HERD will be capable of studying features in the spectra of various nuclei, like single element spectral indices and spectral hardenings/steepenings from hundreds $\mathrm{GeV} /$ nucleon up to hundreds $\mathrm{TeV} /$ nucleon. In particular the proton and helium component could be measured up to PeV energies, thus giving the possibility for the first direct evidence of the knee of the light component due to the reach of maximum energy provided by the source. This result would be fundamental for understanding Galactic CR acceleration/propagation processes.

\section{Gamma Ray Observatory}

\subsection{Interstellar $\gamma$-ray emission from the Galaxy}

The information about cosmic-ray spectra and intensities in distant locations could be extracted by measuring the diffuse $\gamma$-ray emission, that trace energetic particle interactions, primarily protons and electrons, in the ISM [16]. It is relevant for the studies of cosmic-ray acceleration and propagation in the Galaxy [17].

The understanding of the Galactic diffuse emission is also crucial to dark matter studies, since $\gamma$-rays can be produced in cosmic ray interactions mainly via decay of $\pi^{0} \mathrm{~s}$ and possibly from the annihilation of dark matter weakly interacting massive particles (WIMPs) . HERD could measure with high resolution this spectra and indirectly measure the cosmic-ray nucleon and electron spectra, to help disentangle the various contributions. Furthermore, the higher effective area especially at $\mathrm{TeV}$ energies may help in extending the energy range in which we detect the Galactic diffuse emission. This would provide a unique bridge for ground-based experiments sensitive in the $\mathrm{TeV}$ energy range

\subsection{Nature of Dark Matter}

Several fundamental questions should be answered to understand the structure of the Universe at very large scale and its history. These questions are often related to fundamental physics like the possible extension of the standard model of particle physics, quantum gravity, and the fate of antimatter. Gamma-ray observations in the energy band covered by HERD are required to probe several of these questions.

One of the major scientific objectives of a $\gamma$-ray telescope is to identify the possible particle nature of dark matter (DM) by means of the production of secondary $\gamma$-rays after the annihilation (or decay) of the DM particle candidates [18]. The Galactic Center(GC) is expected to be the brightest source of DM annihilations in the $\gamma$-ray sky by several orders of magnitude. Although several astrophysical processes at work in the crowded GC region make it extremely difficult to disentangle the DM signal from conventional emissions, the DM-induced $\gamma$-ray emission is expected to be so large there that its search is of crucial relevance [19]. In the analyses of Fermi data of the Galactic center, the diffuse $\gamma$-ray backgrounds and the discrete sources, as we model them today, seem to account for the majority of the detected $\gamma$-ray emission. Nevertheless, residual emission not accounted for by the above models of standard sources could still be present [20,21] and be interpreted as due to DM annihilation [22]. The spectrum and spatial distribution of the $\mathrm{GeV}$ 
excess is consistent with what can be expected from Weakly Interacting Massive Particle (WIMP) with mass of a few tens of $\mathrm{GeV}$ of annihilating to standard model particles in the inner Galactic halo. With significantly improved sensitivity, HERD could detect and separate additional $\gamma$-ray sources in this region and thus help clarifying the nature of this excess. Furthermore, indirect dark matter search in the $\mathrm{GeV} \gamma$-rays is also complementary to direct search for high mass dark matter candidates, which is not yet well constrained by other means.

Dwarf spheroidal galaxies (dSphs) of the Local Group could also give a clear and unambiguous detection of DM. They are believed to contain up to $10^{3}$ times more DM than visible matter. While their DM-induced gamma-ray luminosity would be smaller than the one of the GC, they are free of standard astrophysical gamma-ray emitters. Their detection in $\gamma$-rays would provide a clear evidence for the presence of WIMPs. In the GeV to TeV WIMP mass range, while no clear evidence for DM signatures has been detected so far, the most robust constraints on the WIMP annihilation crosssection have been obtained from the joint analysis of a dozen of those objects [23]. Considering again an instrument with a better performances in the sub-Tev range would allow probing the inner, most DM dominated, part of the dSphs and minimize the background contamination from diffuse emissions and/or foreground and background sources. Decaying DM produced $\gamma$-ray line might be detectable out of the continuum by HERD [19].

\subsection{Future of multi-messenger astronomy}

The observations in the energy range above $1 \mathrm{GeV}$ by HERD will play a unique and complementary role in multi-wavelength studies across the electromagnetic spectrum with other space and ground telescopes involving radio, optical, X-ray, $\gamma$-ray, and neutrino telescopes. In the event of a nova during the mission, HERD with its large field of view will likely trigger the event and follow the rapid acceleration of CRs in the expansion of the thermonuclear shock wave and ejecta. Fermi data has established novae as $\gamma$-ray emitters and HERD will study the soft $\gamma$-ray spectrum and time profile of the radiating particles to unveil the acceleration mechanisms at play.

The connection between HERD and the Cherenkov Telescope Array (CTA) is extremely useful. CTA will be operative soon (2019/2020) aiming to provide energy coverage from some tens of $\mathrm{GeV}$ to $100 \mathrm{TeV}$. CTA will boost the detection capability of Fermi-LAT by a factor of 25000 in the 20-100 $\mathrm{GeV}$ band, allowing the detection of very short high-energy transients, a completely unexplored territory. It will also substantially improve the angular resolution available in the $\mathrm{TeV}$ and hence the ability to resolve extended sources and their energy dependent morphology. From 2020, the Large High Altitude Air Shower Observatory (LHAASO) will in addition extend the monitored energy range up to $1 \mathrm{PeV}$.

The synergy among HERD, CTA and LHAASO will allow simultaneous coverage of the same sources from few $\mathrm{GeV}$ to $1 \mathrm{PeV}$ with substantial overlap of measured spectra. Only high spatial resolution will allow distinguishing diffuse emission from localized contributions, essential to disentangle acceleration and propagation mechanism in SNRs, PWN, pulsars, and in more extended objects as the Fermi bubbles. Complementary observations with HERD, CTA and LHAASO will also contribute notably to transient science, which is crucial to study for the properties of jets, and can shed light on determining the extragalactic background light, intergalactic magnetic fields, and the validity of the Lorentz invariance. 


\begin{tabular}{ll}
\hline \hline Item & Value \\
\hline Energy range (e/ $\gamma)$ & $10 \mathrm{GeV}-100 \mathrm{TeV} ;>0.1 \mathrm{GeV}(\gamma)$ \\
Energy range (nucleus) & $30 \mathrm{GeV}-3 \mathrm{PeV}$ \\
Angular resolution $(\mathrm{e} / \gamma)$ & $0.1 \mathrm{deg}$ @ $10 \mathrm{GeV}$ \\
Charge measurement (nucleus) & $0.05-0.15 \mathrm{c.u}$. \\
Energy resolution (e) & $1 \% @ 200 \mathrm{GeV}$ \\
Energy resolution (p) & $20 \% @ 100 \mathrm{GeV}-\mathrm{PeV}$ \\
e/p separation & $\sim 10^{-6}$ \\
Geometric factor (e) & $>3 \mathrm{~m}^{2} \mathrm{Sr} @ 200 \mathrm{GeV}$ \\
Geometric factor $(\mathrm{p})$ & $>2 \mathrm{~m}^{2} \mathrm{Sr} @ 100 \mathrm{GeV}$ \\
\hline \hline
\end{tabular}

Table 1: Main requirements of the HERD payload

Gamma-ray observations can be used as a fundamental tool to enhance the discovery potential, in particular by searching for events triggered by gravitational waves or neutrino detection and reciprocally. Advanced Ligo have detected the first signal from a gravitational wave $(\mathrm{GW})$ on 14 September 2015 [24] and together with Virgo they have detected on 17 August 2017 the first GW associated with a Gamma-Ray burst detected by Fermi-GBM [25]. The multi-messenger astrophysics is born and HERD with its unusual large field of view and unique energy coverage, is well suited to the search of electromagnetic counterpart of GW. High-energy neutrino astronomy is also a promising research field entering a new stage in which a discovery of neutrino source might be finally possible with IceCube, and will be further improved with IceCube-Gen2. In the next few years, eROSITA, SVOM, LSST, and Euclid etc. will provide almost all-sky coverage and are efficient transient factories. The synchrotron radio emission $(10 \mathrm{MHz}-1 \mathrm{GHz})$, observed in the current (MWA, LOFAR) or future (SKA) radio surveys, can be used to constrain the spectrum of cosmic-ray electrons with energies of a few $\mathrm{GeV}$. HERD observations of the electron bremsstrahlung emission will complement this spectrum above $\approx 1 \mathrm{GeV}$.

\section{Payload}

In the baseline design, HERD is composed of a 3-D cubic imaging calorimeter (CALO) surrounded by scintillating fiber trackers (FITs) on the top and on the four lateral sides. Then the CALO and FIT are covered by the Silicon Charge Detector (SCD) and the plastic scintillator detector (PSD) from outside. A Transition Radiation Detector (TRD) is located on the lateral side (Fig. 3). SCD is for the accurate measurement of particle absolute charge magnitude $|Z|$. PSD is for trigger of LE gamma and charge measurement; FIT is mainly for particle tracking and charge measurement; CALO is for energy reconstruction and e/p discrimination; TRD is for calibration of $\mathrm{TeV}$ nuclei. The main requirements of the HERD payload are summarized in table 1.

\subsection{CALO}

The CALO [26] is the main detector of HERD instrument: it is a homogeneous, isotropic, 3D segmented calorimeter which can accepts particles coming from each surface. The performance of 


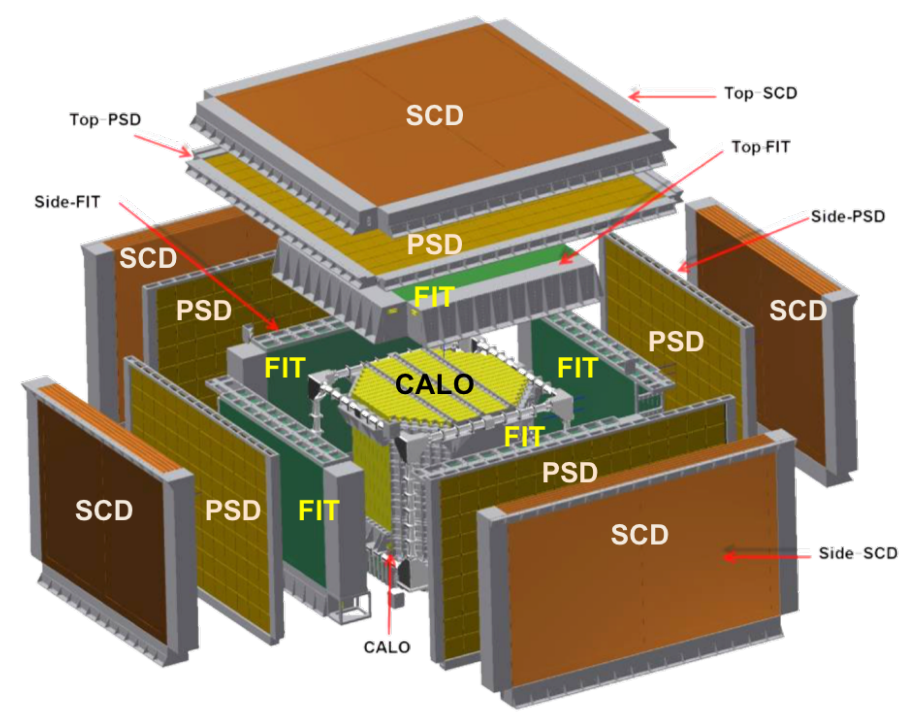

Figure 3: Exploded view of the HERD detector. The innermost subdetector is the calorimeter (CALO), surrounded on the top and the four lateral sides by the scintillating FIber Tracker (FIT), the Plastic Scintillator Detector (PSD) and the Silicon Charge Detector (SCD).

a detector based on these ideas was accurately studied by the CaloCube $R \& D$ project. By exploiting those results, the baseline design of the CALO consists of about 7500 LYSO cubes with edge length of $3 \mathrm{~cm}$, corresponding to about 2.6 radiation lengths $\left(X_{0}\right)$ and 1.4 Molière radius. The CALO external envelope is similar to an octagonal prism and the crystals are arranged on vertical layers with different dimension (fig. 4). The total depth of the CALO for vertical particles is about $55 X_{0}$ and 3 interaction length $\left(\lambda_{I}\right)$.

The scintillation light of each crystal is read-out by two independent systems: the first one consists of WaveLength Shifting fibers (WLS) coupled to image Intensified scientific CMOS (IsCMOS) cameras, the second one is made of photo-diodes (PD) connected to custom front-end electronics chips named HIDRA. This design, here named "double read-out system", achieves the capability of cross-calibrating the scintillation light measurement. Recent articles by calorimetric CR experiments speculated about the energy scale calibration as a possible source of unknown uncertainty, particularly for the electron flux measurement. Both the "double read-out" system and the TRD detector will strongly increase the understanding of HERD calorimeter energy scale with respect to previous CR detectors. Furthermore, both WLS and PD systems provide independent fast trigger information which will be employed to improve the HERD trigger capabilities.

\subsection{FIT}

The HERD scintillating FIber Tracker (FIT) [27] is being designed for the purpose of reconstructing the trajectories and the charge absolute value $(|Z|)$ of charged cosmic rays, favour the conversion of low energy gamma rays and reconstruct the tracks of the generated electrons and positrons.

In the current design, FIT consists of 5 tracking sectors (Fig.5), made of 7 tracking planes, for 7 independent measurements of the position of a traversing charged particle. Each tracking 


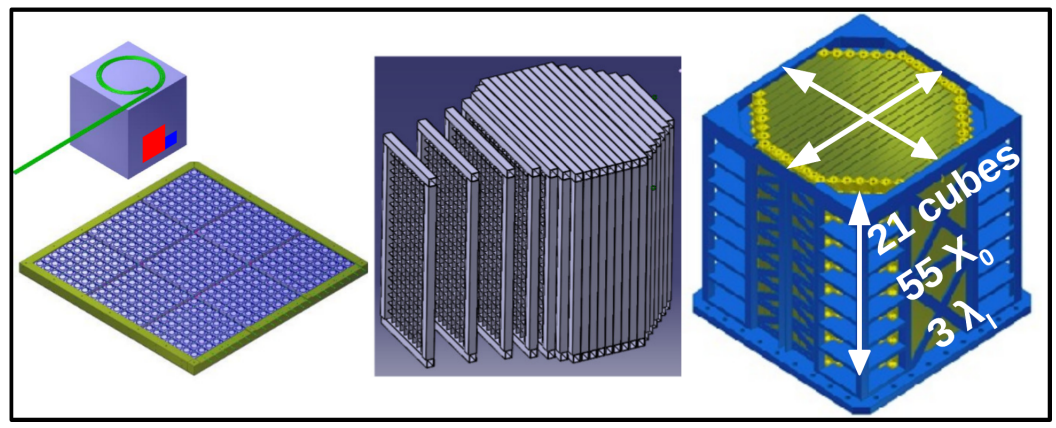

Figure 4: Simplified images of few components of the CALO.

plane consists of two layers of FIT modules measuring the two orthogonal spatial coordinates. The tracking planes of the top sector are made of 10 FIT modules on both the $\mathrm{x}$ layers and y layers, while the tracking planes of the side sectors are made of 6 FIT modules on the $x$ layers and 10 FIT modules on the y layers. A module includes one scintillating fiber mat and three silicon photomultiplier (SiPM) arrays to read out the scintillation light induced by the particles hitting the mat.

A FIT module prototype made of a $77 \mathrm{~cm}$ long fiber mat and one SiPM array was exposed to a $400 \mathrm{GeV} / \mathrm{c}$ primary proton beam and a fragmentation ion beam created by a $150 \mathrm{AGeV} / \mathrm{c}$ lead beam hitting a beryllium target at CERN. A spatial resolution of $45 \mu \mathrm{m}$ and a mean hit efficiency of 99.6\% were measured for protons. The response to nuclei from helium up to beryllium was studied and a charge resolution better than $15 \%$ was found.

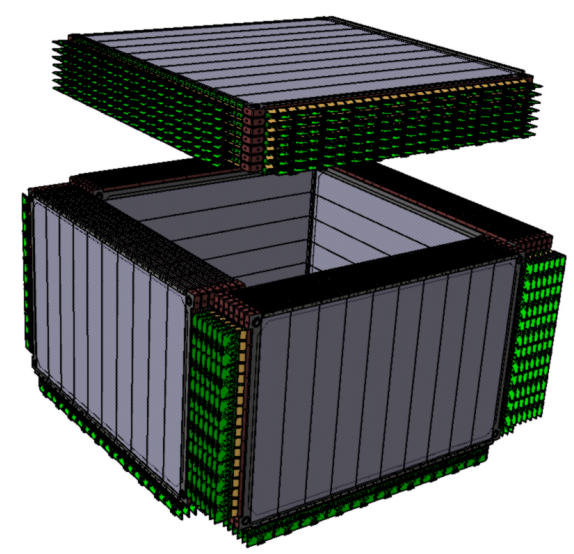

Figure 5: Sketch of the 5-side FIT detector made of 4 lateral sectors and a top sector.

\subsection{PSD}

The PSD of HERD [28] will be utilized as an anti-coincidence detector (discriminating incident photons from charged particles), while providing charge measurement of incoming cosmic-ray nuclei in a range of $Z=1-26$. Organic scintillators are readout by silicon photo-multipliers (SiPMs) instead of conventional PMTs, due to recent technological developments that demonstrate: fast light signal detection; good sensitivity to low light yields; lower power consumption and 
robustness. These features delineate the practicality and versatility of SiPMs in space applications. Moreover, scintillators featuring low density and good radiation hardness, while being affordable and available in mass production, seem as an intriguing choice regarding the PSD realization. Main requirements concerning its design, include: high detection efficiency, broad dynamic range and good energy resolution. Two design layouts are currently investigated, one based on long scintillator bars while the other on square (or rectangular) tiles. Both configurations present advantages and disadvantages, mainly related to the optimal number of readout channels versus back-splash (or back-scattering) effects. Ongoing tests and optimization efforts are carried out for both configurations 6, aiming to define the best scintillator type and size, SiPM model and quantity to be instrumented, along with an overall verification of the PSD configuration, structural robustness and space readiness tests.

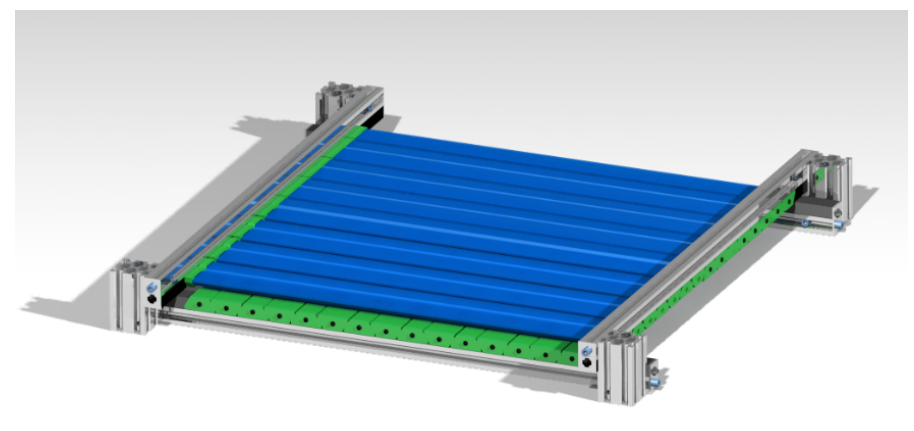

(a)

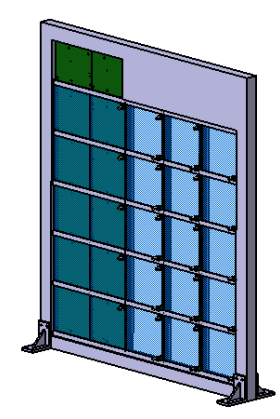

(b)

Figure 6: Preliminary designs of PSD (a) bar and (b) tile prototypes, to be validated via beam tests at CERN.

\subsection{SCD}

The SCD is the first detector encountered by a particle traversing HERD, is placed above the PSD, and is designed to deliver the charge measurement in the HERD wide field of view. The choice of a charge silicon detector system, placed on the top of the instrument, ensures a low level of interactions before and inside the SCD allowing for a reliable charge measurement and minimizing the cosmic-rays nuclei fragmentation reducing the systematics on the HERD flux measurement.

In the current design the SCD is constituted by 5 thin detector units, one squared-shaped with dimensions of $1.6 \times 1.6 \mathrm{~m}^{2}$ placed on the top on the instrument, and 4 with dimensions of $1.4 \times 0.9$ $\mathrm{m}^{2}$ placed on the other 4 sides 7 . Each detection unit contains 8 layers of 300 um microstrip silicon detectors mounted with alternating orthogonal direction strip directions onto low-density aluminum honeycombs. Globally the active area is about $60 \mathrm{~m}^{2}$.

The SCD charge measurement performance has been studied with a MC simulation of the full detector. We simulated several samples of proton, helium, carbon, oxygen, silicon, and iron nuclei with energies from $10 \mathrm{GeV} / \mathrm{n}$ to $1 \mathrm{TeV} / \mathrm{n}$ and with isotropic incidence. We found that the combined charge resolution of 8 layers is below 0.3 c.u. for all samples. Test beams are planned at the CERN SPS ion beam line to directly measure charge resolution of SCD prototypes. 


\section{SCD Simple Design (à la DAMPE)}

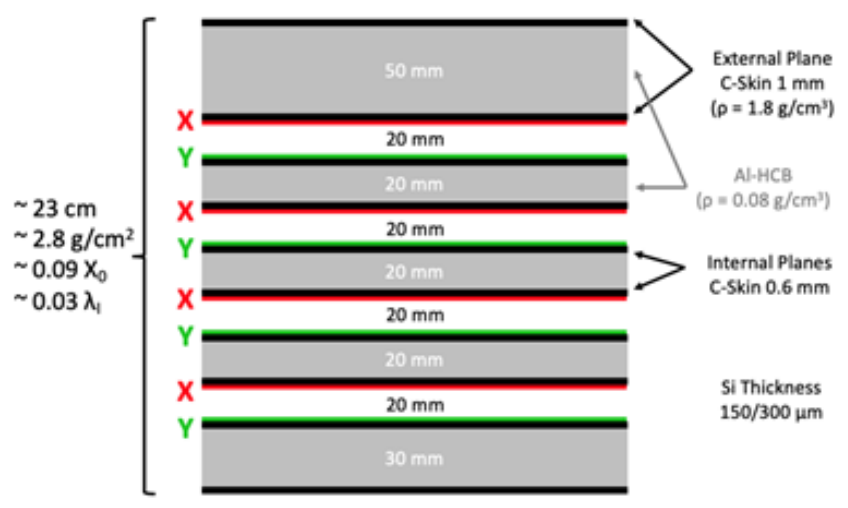

Figure 7: Sketch of the SCD detector.

Design choices for the SCD, as the strip pitch and the distance between SCD and PSD, will also be studied in order to minimize the effect of pile-up in charge measurement due back-splash particle emerging from interactions in the calorimeter. Additionally, SCD is capable of 3D tracking and will be used together with the FIT for the HERD particle track reconstruction. The additional capabilities of the SCD improve the redundancy between different sub-detectors and will allow for cross-calibrations.

\subsection{TRD}

Ground calibration of space calorimeters can only go to $400 \mathrm{GeV}$ at maximum by using CERN SPS beam. For TeV protons, only few particles have showers all contained in the calorimeter and only visible deposited energy is recorded by detectors. Energy of primary proton is then derived by data extrapolation of low energy protons, which is not reliable.

A Transition Radiation Detector (TRD) could be used to calibrate the incident particle with energy in a range in which transition radiation is generated but not saturated. The energy of TR is proportional to the Lorentz factor $\gamma$ of the incident charged particle. The radiant photons will be generated at $\gamma \sim 10^{3}$ and saturated at $\gamma \sim 10^{4}$, the corresponding energy region of proton is about 1-10 TeV. By detecting the $\mathrm{X}$ rays generated by TR, the absolute energy of the incident charged particle in the $\mathrm{TeV}$ region can be obtained by measuring the Lorentz factor.

The large effective area of TRD is achieved by a modular and highly redundant design. The TRD is arranged in 3 layers each consisting of 9 detection modules. Each detection module consists of a TR radiator, an X-ray gaseous detector with an effective area of $20 * 20 \mathrm{~cm}^{2}$ and read-out electronics, supported by grid-like frames.

\section{Conclusion}

The High Energy cosmic-Radiation Detection facility is an international space mission that will start its operation at the end of 2027 on board the future China's Space Station. Thanks to its 
novel design, based on a 3D, homogeneous, isotropic and finely-segmented calorimeter, HERD is expected to accomplish important and frontier goals relative to DM search, CR observations and Gamma-Ray astronomy: It will extend the measurement of $e^{+} e^{-}$flux up to several tens of $\mathrm{TeV}$ testing the hypothesis of the expected cutoff at high energy and helping in distinguish between DM or astrophysical origin of positron excess It will extend the measurement of $\mathrm{p}$ and $\mathrm{He}$ flux up to a few PeV testing the theory of the knee structure as due to acceleration limit Thanks to its large acceptance and high sensitivity to gamma up to several tens of $\mathrm{TeV}$ it will search $\mathrm{g}$ for gamma-line associated to DM annihilation and accomplish a gamma-sky survey up to very high energy.

\section{Acknowledgements}

The author would like to thank all members of the HERD collaboration for supporting this work with valuable comments and fruitful discussions.

\section{References}

[1] L. Farina et al. Gamma-ray performance study of the herd payload. These proceedings 651.

[2] G. Ambrosi et al. Direct detection of a break in the teraelectronvolt cosmic-ray spectrum of electrons and positrons. Nature, 552:63-66, 2017.

[3] Qiang Yuan, Xiao-Jun Bi, Guo-Ming Chen, Yi-Qing Guo, Su-Jie Lin, and Xinmin Zhang. Implications of the AMS-02 positron fraction in cosmic rays. Astropart. Phys., 60:1-12, 2015.

[4] Su-Jie Lin, Qiang Yuan, and Xiao-Jun Bi. Quantitative study of the AMS-02 electron/positron spectra: Implications for pulsars and dark matter properties. Phys. Rev., D91(6):063508, 2015.

[5] Kun Fang, Xiao-Jun Bi, and Peng-Fei Yin. Explanation of the knee-like feature in the DAMPE cosmic $e^{-}+e^{+}$energy spectrum. Astrophys. J., 854(1):57, 2018.

[6] Kun Fang, Bing-Bing Wang, Xiao-Jun Bi, Su-Jie Lin, and Peng-Fei Yin. Perspective on the cosmic-ray electron spectrum above TeV. Astrophys. J., 836(2):172, 2017.

[7] D. J. Bird et al. Detection of a cosmic ray with measured energy well beyond the expected spectral cutoff due to cosmic microwave radiation. Astrophys. J., 441:144-150, 1995.

[8] Johannes Blumer, Ralph Engel, and Jorg R. Horandel. Cosmic Rays from the Knee to the Highest Energies. Prog. Part. Nucl. Phys., 63:293-338, 2009.

[9] Jorg R. Hoerandel. Models of the knee in the energy spectrum of cosmic rays. Astropart. Phys., 21:241-265, 2004.

[10] Mirko Boezio and Emiliano Mocchiutti. Chemical Composition of Galactic Cosmic Rays with Space Experiments. Astropart. Phys., 39-40:95-108, 2012.

[11] E. S. Seo. Direct measurements of cosmic rays using balloon borne experiments. Astropart. Phys., 39-40:76-87, 2012. 
[12] H. S. Ahn et al. Discrepant hardening observed in cosmic-ray elemental spectra. Astrophys. J., 714:L89-L93, 2010.

[13] Y. S. Yoon et al. Cosmic-Ray Proton and Helium Spectra from the First CREAM Flight. Astrophys. J., 728:122, 2011.

[14] Karl-Heinz Kampert and Michael Unger. Measurements of the Cosmic Ray Composition with Air Shower Experiments. Astropart. Phys., 35:660-678, 2012.

[15] B. Bartoli et al. Knee of the cosmic hydrogen and helium spectrum below $1 \mathrm{PeV}$ measured by ARGO-YBJ and a Cherenkov telescope of LHAASO. Phys. Rev., D92(9):092005, 2015.

[16] Stanley D Hunter, DL Bertsch, JR Catelli, TM Dame, SW Digel, BL Dingus, JA Esposito, CE Fichtel, RC Hartman, G Kanbach, et al. EGRET observations of the diffuse gamma-ray emission from the galactic plane. The Astrophysical Journal, 481(1):205, 1997.

[17] Igor V Moskalenko, Andrew W Strong, and Stepan G Mashnik. Propagation of Cosmic Rays: Nuclear Physics in Cosmic-Ray Studies. In AIP Conference Proceedings, volume 769, pages 1612-1617. AIP, 2005.

[18] Lars Bergström, Piero Ullio, and James H Buckley. Observability of $\gamma$ rays from dark matter neutralino annihilations in the Milky Way halo. Astroparticle Physics, 9(2):137-162, 1998.

[19] Germán A Gómez-Vargas, Miguel A Sánchez-Conde, Ji-Haeng Huh, Miguel Peiró, Francisco Prada, Aldo Morselli, Anatoly Klypin, David G Cerdeño, Yann Mambrini, and Carlos Muñoz. Constraints on WIMP annihilation for contracted dark matter in the inner Galaxy with the Fermi-LAT. Journal of Cosmology and Astroparticle Physics, 2013(10):029, 2013.

[20] Vincenzo Vitale, Aldo Morselli, et al. Indirect search for dark matter from the center of the milky way with the Fermi-Large Area Telescope. arXiv:0912.3828, 2009.

[21] Bei Zhou, Yun-Feng Liang, Xiaoyuan Huang, Xiang Li, Yi-Zhong Fan, Lei Feng, and Jin Chang. GeV excess in the Milky Way: The role of diffuse galactic gamma-ray emission templates. Physical Review D, 91(12):123010, 2015.

[22] Abraham Achterberg, Simone Amoroso, Sascha Caron, Luc Hendriks, Roberto Ruiz De Austri, and Christoph Weniger. A description of the Galactic Center excess in the Minimal Supersymmetric Standard Model. Journal of Cosmology and Astroparticle Physics, 2015(08):006, 2015.

[23] Markus Ackermann, A Albert, Brandon Anderson, L Baldini, J Ballet, G Barbiellini, D Bastieri, K Bechtol, R Bellazzini, E Bissaldi, et al. Dark matter constraints from observations of 25 Milky Way satellite galaxies with the Fermi Large Area Telescope. Physical Review D, 89(4):042001, 2014.

[24] B. P. Abbott et al. Observation of gravitational waves from a binary black hole merger. Physical Review Letters, 116:061102, Feb 2016. 
[25] B. P. Abbott et al. Gravitational Waves and Gamma-rays from a Binary Neutron Star Merger: GW170817 and GRB 170817A. The Astrophysical Journal, 848(2):L13, 2017.

[26] L. Pacini et al. Design and expected performances of the large acceptance calorimeter for the herd space mission. These proceedings 066.

[27] C. Perrina et al. Fit: the scintillating fiber tracker of the herd space mission. These proceedings 067.

[28] D. Kyratzis et al. The plastic scintillator detector of the herd space mission. These proceedings 054. 


\section{Full Authors List: HERD Collaboration}

O. Adriani ${ }^{26}$, F. Alemanno ${ }^{27}$, R. Aloisio ${ }^{27}$, C. Altomare ${ }^{23}$, G. Ambrosi ${ }^{34}$, Q. An ${ }^{10}$, M. Antonelli ${ }^{45}$, P. Azzarello ${ }^{37}$, L. Bai ${ }^{8}$, Y.L. Bai ${ }^{3}$, T.W. Bao ${ }^{1}$, M. Barbanera ${ }^{34}$, F.C.T. Barbato ${ }^{27}$, P. Bernardini ${ }^{30}$, E. Berti ${ }^{26}$, B. Bertucci ${ }^{35}$, X.J. Bi ${ }^{1}$, G. Bigongiari ${ }^{36}$, M. Bongi ${ }^{26}$, V. Bonvicini $^{45}$, P. Bordas ${ }^{40}$, V. Bosch-Ramon ${ }^{40}$, S. Bottai ${ }^{25}$, P. Brogi ${ }^{36}$, F. Cadoux ${ }^{37}$, D. Campana ${ }^{31}$, W.W. Cao ${ }^{3}$, Z. Cao ${ }^{1}$, J. Casaus ${ }^{39}$, E. Catanzani ${ }^{35}$, P. W. Cattaneo ${ }^{33}$, J. Chang ${ }^{9,13}$, Y.H. Chang ${ }^{21}$, G.M. Chen ${ }^{1}$, Y. Chen ${ }^{15}$, F. Cianetti ${ }^{35}$, A. Comerma ${ }^{40,41}$, D. Cortis ${ }^{28}$, X.H. Cui ${ }^{13}$, X.Z. Cui ${ }^{1}$, C. Dai ${ }^{5}$, Z.G. Dai ${ }^{15}$, R. D’Alessandro ${ }^{26}$, S. De Gaetanoe ${ }^{24}$, I. De Mitri ${ }^{27}$, F. de Palma ${ }^{30}$, V. Di Felice ${ }^{50}$, A. Di Giovanni ${ }^{27}$, M. Di Santo ${ }^{27}$, L. Di Venere ${ }^{24}$, J.N. Dong ${ }^{6,7}$, Y.W. Dong ${ }^{1}$, G. Donvito ${ }^{23}$, M. Duranti ${ }^{34}$, D. D’Urso ${ }^{49}$, C. Evoli ${ }^{27}$, K. Fang ${ }^{1}$, L. Fariña ${ }^{42}$, Y. Favre ${ }^{37}$, C.Q. Feng ${ }^{10}$, H. Feng ${ }^{16}$, H.B. Feng ${ }^{5}$, Z.K. Feng ${ }^{5}$, N. Finetti ${ }^{22}$, V. Formato ${ }^{50}$, J. M. Frieden ${ }^{44}$, P. Fusco $^{24}$, J.R. Gao ${ }^{3}$, F. Gargano ${ }^{23}$, D. Gascon-Fora ${ }^{40}$, D. Gasparrini ${ }^{50}$, N. Giglietto ${ }^{24}$, F. Giovacchini ${ }^{39}$, S. Gomez ${ }^{40}$, K. Gong ${ }^{1}$, Q.B. $\mathrm{Gou}^{1}$, R. Guida ${ }^{46}$, D.Y. Guo ${ }^{1}$, J.H. Guo ${ }^{9}$, Y.Q. Guo ${ }^{1}$, H.H. He ${ }^{1}$, H.B. Hu${ }^{1}$, J.Y. Hu ${ }^{1}, 2$, P. Hu ${ }^{1,2}$, Y.M. Hu ${ }^{9}$, G.S. Huang ${ }^{10}$, J. Huang ${ }^{1}$, W.H. Huang ${ }^{6,7}$, X.T. Huang ${ }^{6,7}$, Y.B. Huang ${ }^{5}$, Y.F. Huang ${ }^{15}$, M. Ionica ${ }^{34}$, L. Jouvin ${ }^{42}$, A. Kotenko ${ }^{37}$, D. Kyratzis ${ }^{27}$, D. La Marra ${ }^{37}$, M.J. Li ${ }^{6,7}$, Q.Y. Li $\mathrm{Li}^{6,7}$, R. Li ${ }^{3}$, S.L. Li ${ }^{1,2}$, T. Li ${ }^{6,7}$, X. Li ${ }^{9}$, Z. Li ${ }^{17}$, Z.H. Li ${ }^{1,2}$, E.W. Liang , M.J. Liang ${ }^{1,2}$, C.L. Liao ${ }^{8}$, F. Licciulli ${ }^{23}$,

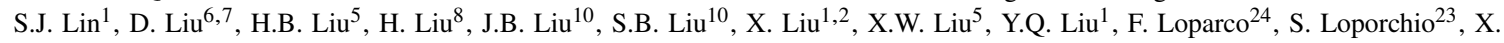
$\mathrm{Lu}^{5}$, J.G. Lyu ${ }^{4}$, L.W. Lyu ${ }^{3}$, P. Maestro ${ }^{36}$, E. Mancini ${ }^{34}$, R. Manera ${ }^{40}$, J. Marin ${ }^{39}$, P. S. Marrocchesi ${ }^{36}$, G. Marsella ${ }^{53}$, G. Martinez ${ }^{39}$, M. Martinez ${ }^{42}$, D. Marzullo ${ }^{47}$, J. Mauricio ${ }^{40}$, E. Mocchiutti4 ${ }^{45}$, G. Morettini ${ }^{35}$, N. Mori' ${ }^{25}$, L. Mussolin ${ }^{35}$, M. Nicola Mazziotta ${ }^{23}$, A. Oliva $^{51}$, D. Orlandi ${ }^{28}$, G. Osteria ${ }^{31}$, L. Pacini ${ }^{25}$, B. Panico ${ }^{31}$, F.R. Pantalei ${ }^{24}$, S. Papa ${ }^{46}$, P. Papini ${ }^{25}$, J.M. Paredes ${ }^{40}$, A. Parenti ${ }^{27}$, M.

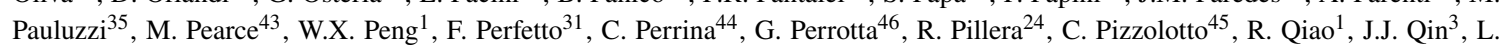

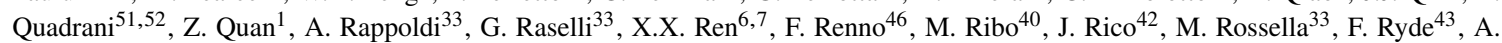
$\mathrm{Sanmukh}^{40}$, V. Scotti ${ }^{32}$, D. Serini ${ }^{23}$, D.L. Shi ${ }^{3}$, Q.Q. Shi ${ }^{6,7}$, L. Silveri2 ${ }^{27}$, O. Starodubtsev ${ }^{25}$, D.T. Su ${ }^{12}$, M. Su ${ }^{20}$, D. Sukhonos ${ }^{37}$, A. $\mathrm{Suma}^{46}$, X.L. Sun ${ }^{1}$, Z.T. Sun ${ }^{1,2}$, A. Surdo ${ }^{29}$, Z.C. Tang ${ }^{1}$, A. Tiberio ${ }^{26}$, A. Tykhonov ${ }^{37}$, V. Vagelli ${ }^{48}$, E. Vannuccini ${ }^{25}$, M. Velasco ${ }^{39}$, R. Walter ${ }^{38}$, A.Q. Wang ${ }^{6,7}$, B. Wang ${ }^{3}$, J.C. Wang ${ }^{14}$, J.M. Wang ${ }^{1}$, J.J. Wang ${ }^{1,2}$, L. Wang ${ }^{13}$, M. Wang ${ }^{6,7}$, R.J. Wang ${ }^{1}$, S. Wang ${ }^{9}$, X.Y. $\mathrm{Wang}^{15}$, X.L Wang ${ }^{10}$, Z.G. Wang ${ }^{1}$, D.M. Wei ${ }^{9}$, J.J. Wei ${ }^{9}$, B.B. Wu ${ }^{1}$, J. Wu ${ }^{19}$, L.B. Wu ${ }^{27}$, X. Wu ${ }^{37}$, X.F. Wu ${ }^{9}$, Y.L. Xin ${ }^{8}$, M. Xu ${ }^{1}$, Z.Z. $\mathrm{Xu}^{10}$, H.R. Yan ${ }^{17}$, Y. Yang ${ }^{3}$, P.F. Yin ${ }^{1}$, Y.W. Yu ${ }^{18}$, Q. Yuan ${ }^{9}$, G. Zampa ${ }^{45}$, N. Zampa ${ }^{45}$, M. Zha ${ }^{1}$, C. Zhang ${ }^{1}$, F.Z. Zhang ${ }^{1,2}$, L. Zhang ${ }^{1}$, L. Zhang ${ }^{1}$, L.F. Zhang ${ }^{1,2}$, S.N. Zhang ${ }^{1,2}$, Y. Zhang ${ }^{9}$, Y.L. Zhang ${ }^{10}$, Z.G. Zhao ${ }^{10}$, J.K. Zheng ${ }^{3}$, Y.L. Zhou ${ }^{5}$, F.R. Zhu ${ }^{8}$, and K.J. Zhu ${ }^{4}$

1 Key Laboratory of Particle and Astrophysics, Chinese Academy of Sciences, Beijing, China

2 University of Chinese Academy of Sciences, Beijing, China

Xi' an Institute of Optics and Precision Mechanics of CAS, Xi' an, China

Institute of High Energy Physics, Chinese Academy of Sciences, Beijing, China

School of Physical Science and Technology, Guangxi University, Nanning, China

Institute of Frontier and Interdisciplinary Science, Shandong University, Qingdao, China

Key Laboratory of Particle Physics and Particle Irradiation, Ministry of Education, China

School of Physical Science and Technology, Southwest Jiaotong University, Chengdu, China

Key Laboratory of Dark Matter and Space Astronomy, Purple Mountain Observatory, Chinese Academy of Sciences, Nanjing 210023, China

10 Department of Modern Physics, University of Science and Technology of China, Hefei, China

11 Department of Astronomy, Yunan University, Kunming, China

12 North Night Vision Technology co. 1td., Kunming, China

13 National Astronomical Observatories, Chinese Academy of Sciences, Beijing 100101, China

14 Yunnan Astronomical Observatory, Chinese Academy of Sciences, Kunming, China

15 School of Astronomy and Space Science, Nanjing University, Nanjing, China

16 Department of Astronomy, Tsinghua University, Beijing, China

17 Department of Astronomy, Peking University, Beijing, China

18 Institute of Astrophysics, Central China Normal University, Wuhan, China

19 Department of Physics, China University of Geosciences, Wuhan, China

20 The University of Hong Kong, Hong Kong, China

21 Institute of Physics, Academia Sinica, Taipei, Taiwan

22 Universitá dell'Aquila and Istituto Nazionale di Fisica Nucleare, Sezione di Firenze, Firenze, Italy

23 Istituto Nazionale di Fisica Nucleare, Sezione di Bari, Bari, Italy

24 Dipartimento di Fisica "M.Merlin" dell?Univerisitá e del Politecnico di Bari, and Istituto Nazionale di Fisica Nucleare, Sezione di Bari, Bari, Italy

25 Istituto Nazionale di Fisica Nucleare, Sezione di Firenze, Firenze, Italy

26 Universitá di Firenze and Istituto Nazionale di Fisica Nucleare, Sezione di Firenze, Firenze, Italy

27 Gran Sasso Science Institute, L'Aquila, Italy and INFN Laboratori Nazionali del Gran Sasso, Assergi, L’Aquila, Italy

28 INFN Laboratori Nazionali del Gran Sasso, L'Aquila , Italy

29 Istituto Nazionale di Fisica Nucleare, Sezione di Lecce, Lecce, Italy

30 Universitá del Salento and Istituto Nazionale di Fisica Nucleare, Sezione di Lecce, Lecce, Italy

31 Istituto Nazionale di Fisica Nucleare, Sezione di Napoli, Napoli, Italy

32 Universitá di Napoli "Federico II" and Istituto Nazionale di Fisica Nucleare, Sezione di Napoli, Napoli, Italy

33 Istituto Nazionale di Fisica Nucleare, Sezione di Pavia, Pavia, Italy 
34 Istituto Nazionale di Fisica Nucleare, Sezione di Perugia, Perugia, Italy

35 Universitá degli Studi di Perugia and Istituto Nazionale di Fisica Nucleare, Sezione di Perugia, Perugia, Italy

36 Universitá di Siena and Istituto Nazionale di Fisica Nucleare, Sezione di Pisa, Pisa, Italy

37 Département de Physique Nucléaire et Corpusculaire (DPNC), Université de Genève, Genève, Switzerland

38 Department of Astronomy, University of Geneva, Geneva, Switzerland

39 Centro de Investigaciones Energéticas, Medioambientales y Tecnoló gicas (CIEMAT), E-28040 Madrid, Spain

40 Dept. Física Quàntica i Astrofísica, Institut de Ciències del Cosmos (ICCUB), Universitat de Barcelona (IEEC-UB), Barcelona, Spain

41 Universitat Pompeu Fabra (UPF), Barcelona, Spain

42 Institut de Física d'Altes Energies (IFAE), The Barcelona Institute of Science and Technology (BIST), E-08193 Bellaterra, Barcelona, Spain

43 KTH Royal Institute of Technology, Stockholm, Sweden

44 Institute of Physics, Ecole Polytechnique Fédérale de Lausanne (EPFL), Lausanne, Switzerland

45 Istituto Nazionale di Fisica Nucleare, Sezione di Trieste, Trieste, Italy

46 Universitá degli Studi di Napoli Federico II, Napoli, Italy

47 Universitá degli Studi di Trieste, Italy

48 Italian Space Agency and Istituto Nazionale di Fisica Nucleare, Sezione di Perugia, Perugia, Italy

49 Universitá degli Studi di Sassari and Istituto Nazionale di Fisica Nucleare, Sezione di Perugia, Perugia, Italy

50 Istituto Nazionale di Fisica Nucleare, Sezione di Roma Tor Vergata, Rome, Italy

51 INFN Sezione di Bologna, 40126 Bologna, Italy

52 INFN Sezione di Bologna, 40126 Bologna, Italy; Universitá di Bologna, 40126 Bologna, Italy

53 Dipartimento di Fisica e Chimica "E. Segrè" Universitá degli Studi di Palermo, Palermo, Italy and INFN sez. Catania, Catania, Italy 